\title{
On the two-dimensional theta functions of the Borweins
}

\author{
by
}

Ayşe Alaca, Şaban Alaca and Kenneth S. Williams (Ottawa)

1. Introduction. Let $\mathbb{N}_{0}, \mathbb{N}, \mathbb{Z}, \mathbb{R}$ and $\mathbb{C}$ denote the sets of nonnegative integers, positive integers, integers, real numbers and complex numbers respectively. Jonathan and Peter Borwein [4], in their work on a cubic counterpart of Jacobi's theta function identity and a cubic analogue of the arithmetic-geometric mean iteration of Gauss and Legendre, introduced the following three 2-dimensional theta functions:

$$
\begin{aligned}
a(q) & :=\sum_{(m, n) \in \mathbb{Z}^{2}} q^{m^{2}+m n+n^{2}}, \quad q \in \mathbb{C},|q|<1, \\
b(q) & :=\sum_{(m, n) \in \mathbb{Z}^{2}} \omega^{m-n} q^{m^{2}+m n+n^{2}}, \quad q \in \mathbb{C},|q|<1, \\
c(q) & :=\sum_{(m, n) \in \mathbb{Z}^{2}} q^{(m+1 / 3)^{2}+(m+1 / 3)(n+1 / 3)+(n+1 / 3)^{2}}, \quad q \in \mathbb{C},|q|<1,
\end{aligned}
$$

where $\omega=e^{2 \pi i / 3}$. Note that in (1.3) principal values of the cube roots are taken. We observe that

$$
a(0)=1, \quad b(0)=1, \quad c(0)=0 .
$$

The Borweins [4] (and together with F. G. Garvan [5]) proved the beautiful cubic identity

$$
a(q)^{3}=b(q)^{3}+c(q)^{3} .
$$

The Jacobi theta function $\varphi(q)$ is defined by

$$
\varphi(q):=\sum_{n=-\infty}^{\infty} q^{n^{2}}, \quad q \in \mathbb{C},|q|<1 .
$$

2000 Mathematics Subject Classification: 11D45, 11E20, 11E25, 11F27.

Key words and phrases: theta functions, Eisenstein series, quaternary forms.

Research of the third author was supported by Natural Sciences and Engineering Research Council of Canada grant A-7233. 
We have

$$
\varphi(0)=1
$$

Set

$$
\begin{aligned}
& p(q):=\frac{\varphi^{2}(q)-\varphi^{2}\left(q^{3}\right)}{2 \varphi^{2}\left(q^{3}\right)} \\
& k(q):=\frac{\varphi^{3}\left(q^{3}\right)}{\varphi(q)}
\end{aligned}
$$

Clearly

$$
p(0)=0, \quad k(0)=1 .
$$

When there is no risk of confusion we write $p$ for $p(q)$ and $k$ for $k(q)$. Making use of identities proved in [1], [2] and [5], we prove the following parametric representations of $a\left(q^{m}\right), b\left(q^{m}\right), c\left(q^{m}\right)(m \in\{1,2,3,4,6,8,12\})$, as well as of $a(-q), b(-q), c(-q)$, in terms of $p$ and $k$. Since $b\left(q^{m}\right)$ can be determined from $a\left(q^{m}\right)$ and $c\left(q^{m}\right)$ by means of (1.5) (with $q$ replaced by $q^{m}$ ), we only give the values of $b\left(q^{m}\right)$ when they can be expressed in terms of $p$ and $k$ in a fairly simple manner.

THEOREM 1.

$$
\begin{aligned}
& a(q)=\left(1+4 p+p^{2}\right) k, \\
& b(q)=2^{-1 / 3}\left((1-p)^{4}(1+2 p)(2+p)\right)^{1 / 3} k, \\
& c(q)=2^{-1 / 3} 3\left(p(1+p)^{4}\right)^{1 / 3} k .
\end{aligned}
$$

THEOREM 2.

$$
\begin{aligned}
& a\left(q^{2}\right)=\left(1+p+p^{2}\right) k \\
& b\left(q^{2}\right)=2^{-2 / 3}((1-p)(1+2 p)(2+p))^{2 / 3} k \\
& c\left(q^{2}\right)=2^{-2 / 3} 3(p(1+p))^{2 / 3} k
\end{aligned}
$$

Theorem 3.

$$
\begin{aligned}
& a\left(q^{3}\right)=3^{-1}\left(1+4 p+p^{2}+2^{2 / 3}\left((1-p)^{4}(1+2 p)(2+p)\right)^{1 / 3}\right) k \\
& c\left(q^{3}\right)=3^{-1}\left(1+4 p+p^{2}-2^{-1 / 3}\left((1-p)^{4}(1+2 p)(2+p)\right)^{1 / 3}\right) k .
\end{aligned}
$$

TheOrem 4.

$$
\begin{aligned}
& a\left(q^{4}\right)=\left(1+p-\frac{1}{2} p^{2}\right) k \\
& b\left(q^{4}\right)=2^{-4 / 3}\left((1-p)(1+2 p)(2+p)^{4}\right)^{1 / 3} k \\
& c\left(q^{4}\right)=2^{-4 / 3} 3\left(p^{4}(1+p)\right)^{1 / 3} k
\end{aligned}
$$

Theorem 5.

$$
\begin{aligned}
& a\left(q^{6}\right)=3^{-1}\left(1+p+p^{2}+2^{1 / 3}((1-p)(1+2 p)(2+p))^{2 / 3}\right) k \\
& c\left(q^{6}\right)=3^{-1}\left(1+p+p^{2}-2^{-2 / 3}((1-p)(1+2 p)(2+p))^{2 / 3}\right) k
\end{aligned}
$$


Theorem 6.

$$
a\left(q^{8}\right)=2^{-2}\left(1+p+p^{2}+3((1-p)(1+p)(1+2 p))^{1 / 2}\right) k .
$$

Theorems $1-5$ are proved in Sections 2-6 respectively. Theorem 6 is proved in Section 12 by applying the "duplication principle" (Theorem 9) to Theorem 4 . We omit the complicated expressions for $b\left(q^{8}\right)$ and $c\left(q^{8}\right)$. We could also determine $a\left(q^{9}\right)$ and $c\left(q^{9}\right)$ by applying the "triplication principle" (Theorem 10) to Theorem 3. However the resulting expressions for $a\left(q^{9}\right)$ and $c\left(q^{9}\right)$ are complicated so we do not give them here.

TheOREM 7.

$$
\begin{aligned}
& a\left(q^{12}\right)=3^{-1}\left(1+p-\frac{1}{2} p^{2}+2^{-1 / 3}\left((1-p)(1+2 p)(2+p)^{4}\right)^{1 / 3}\right) k, \\
& c\left(q^{12}\right)=3^{-1}\left(1+p-\frac{1}{2} p^{2}-2^{-4 / 3}\left((1-p)(1+2 p)(2+p)^{4}\right)^{1 / 3}\right) k .
\end{aligned}
$$

Theorem 7 is proved in Section 7. Alternative proofs can be given by applying the duplication principle to Theorem 5 and by applying the triplication principle to Theorem 4 .

THEOREM 8.

$$
\begin{aligned}
& a(-q)=\left(1-2 p-2 p^{2}\right) k, \\
& b(-q)=2^{-1 / 3}\left((1-p)(1+2 p)^{4}(2+p)\right)^{1 / 3} k, \\
& c(-q)=-2^{1 / 3} 3(p(1+p))^{1 / 3} k .
\end{aligned}
$$

Theorem 8 is proved in Section 8.

From Theorems 1, 2 and 4, we obtain the duplication principle for $p$ and $k$, namely

Theorem 9 (Duplication principle).

$$
\begin{aligned}
& p\left(q^{2}\right)=\frac{1+p-p^{2}-((1-p)(1+p)(1+2 p))^{1 / 2}}{p^{2}}, \\
& k\left(q^{2}\right)=\frac{\left(1+p-p^{2}+((1-p)(1+p)(1+2 p))^{1 / 2}\right) k}{2} .
\end{aligned}
$$

Theorem 9 is proved in Section 9.

From Theorems 1, 2, 3 and 5, we obtain the triplication principle for $p$ and $k$, namely

Theorem 10 (Triplication principle).

$$
\begin{aligned}
p\left(q^{3}\right)= & 3^{-1}\left(\left(-4-3 p+6 p^{2}+4 p^{3}\right)\right. \\
& +2^{2 / 3}\left(1-2 p-2 p^{2}\right)((1-p)(1+2 p)(2+p))^{1 / 3} \\
& \left.+2^{1 / 3}(1+2 p)((1-p)(1+2 p)(2+p))^{2 / 3}\right), \\
k\left(q^{3}\right)= & 3^{-2}\left(3+2^{2 / 3}(1+2 p)((1-p)(1+2 p)(2+p))^{1 / 3}\right. \\
& \left.+2^{4 / 3}((1-p)(1+2 p)(2+p))^{2 / 3}\right) k .
\end{aligned}
$$


Theorem 10 is proved in Section 11.

From Theorems 1 and 9, we obtain the "change of sign principle" for $p$ and $k$, namely

Theorem 11 (Change of sign principle).

$$
p(-q)=\frac{-p}{1+p}, \quad k(-q)=(1+p)^{2} k .
$$

Theorem 11 is proved in Section 10 .

For $n \in \mathbb{N}_{0}$ and $l, m \in \mathbb{N}$ we set

$$
\begin{aligned}
& N(l, m ; n) \\
& \quad:=\operatorname{card}\left\{(x, y, z, t) \in \mathbb{Z}^{4} \mid l\left(x^{2}+x y+y^{2}\right)+m\left(z^{2}+z t+t^{2}\right)=n\right\} .
\end{aligned}
$$

Clearly $N(l, m ; 0)=1$ and

$$
\sum_{n=0}^{\infty} N(l, m ; n) q^{n}=a\left(q^{l}\right) a\left(q^{m}\right) .
$$

As an application of Theorems $1-7$, we determine $N(l, m ; n)$ for certain small values of $l$ and $m$. In preparation for doing this we prove in Sections 13-15 some results concerning the Eisenstein series

$$
L(q)=1-24 \sum_{n=1}^{\infty} \sigma(n) q^{n},
$$

where

$$
\sigma(m)= \begin{cases}\sum_{d \mid m} d & \text { if } m \in \mathbb{N}, \\ 0 & \text { if } m \notin \mathbb{N} .\end{cases}
$$

Theorem 12. For $n \in \mathbb{N}$,

$$
N(1,1 ; n)=12 \sigma(n)-36 \sigma(n / 3) .
$$

The proof of Theorem 12 is given in Section 16. A result equivalent to Theorem 12 was stated but not proved by Liouville [7]. An elementary proof was given by Huard, Ou, Spearman and Williams [6].

Theorem 13. For $n \in \mathbb{N}$,

$$
N(1,2 ; n)=6 \sigma(n)-12 \sigma(n / 2)+18 \sigma(n / 3)-36 \sigma(n / 6) .
$$

The proof of Theorem 13 is given in Section 17. Liouville [8] gave a result equivalent to Theorem 13 but did not prove it.

Theorem 14. For $n \in \mathbb{N}$,

$$
N(1,3 ; n)= \begin{cases}12 \sigma(n)-36 \sigma(n / 3) & \text { if } n \equiv 0(\bmod 3), \\ 6 \sigma(n) & \text { if } n \equiv 1(\bmod 3), \\ 0 & \text { if } n \equiv 2(\bmod 3) .\end{cases}
$$


The proof of Theorem 14 is given in Section 18. Liouville [11] stated a result equivalent to Theorem 14 but did not prove it.

Theorem 15. For $n \in \mathbb{N}$,

$$
N(1,4 ; n)= \begin{cases}12 \sigma(n / 4)-36 \sigma(n / 12) & \text { if } n \equiv 0(\bmod 2), \\ 6 \sigma(n)-18 \sigma(n / 3) & \text { if } n \equiv 1(\bmod 2) .\end{cases}
$$

The proof of Theorem 15 is given in Section 19. Liouville did not consider the evaluation of $N(1,4 ; n)$ and the result appears to be new.

TheOREM 16. For $n \in \mathbb{N}$,

$$
N(1,6 ; n)= \begin{cases}-6 \sigma(n)+12 \sigma(n / 2)+30 \sigma(n / 3)-60 \sigma(n / 6) & \text { if } n \equiv 0(\bmod 3), \\ 6 \sigma(n)-12 \sigma(n / 2) & \text { if } n \equiv 1(\bmod 3), \\ 0 & \text { if } n \equiv 2(\bmod 3) .\end{cases}
$$

Theorem 16 is proved in Section 20. Liouville [9] stated but did not prove a result equivalent to Theorem 16 .

Theorem 17. For $n \in \mathbb{N}$,

$$
N(2,3 ; n)= \begin{cases}-6 \sigma(n)+12 \sigma(n / 2)+30 \sigma(n / 3)-60 \sigma(n / 6) & \text { if } n \equiv 0(\bmod 3), \\ 0 & \text { if } n \equiv 1(\bmod 3), \\ 6 \sigma(n)-12 \sigma(n / 2) & \text { if } n \equiv 2(\bmod 3) .\end{cases}
$$

Theorem 17 is proved in Section 21. Liouville [10] stated but did not prove a result equivalent to Theorem 17.

We close our introduction by noting that for small $|q|$ we have

$$
\begin{aligned}
& a(q)=1+6 q+O\left(q^{2}\right), \\
& b(q)=1-3 q+O\left(q^{2}\right), \\
& c(q)=3 q^{1 / 3}\left(1+q+O\left(q^{2}\right)\right) \\
& \varphi(q)=1+2 q+O\left(q^{4}\right) \\
& p(q)=2 q+2 q^{2}+O\left(q^{3}\right), \\
& k(q)=1-2 q+O\left(q^{3}\right) .
\end{aligned}
$$

Hence

$$
\frac{c\left(q^{2}\right)}{c^{2}(q)}=\frac{3 q^{2 / 3}\left(1+O\left(q^{2}\right)\right)}{9 q^{2 / 3}(1+O(q))}=\frac{1}{3}(1+O(q))
$$

so

$$
\lim _{q \rightarrow 0^{+}} \frac{c\left(q^{2}\right)}{c^{2}(q)}=\frac{1}{3}
$$


2. Proof of Theorem 1. For $n \in \mathbb{N}$ set

$$
z_{n}=z_{n}(q)=\varphi^{2}\left(q^{n}\right)
$$

Also set

$$
m=m(q)=\frac{z_{1}}{z_{3}}=\frac{\varphi^{2}(q)}{\varphi^{2}\left(q^{3}\right)} .
$$

From (1.8) and (2.2) we deduce

$$
m=2 p+1 .
$$

From (1.9), (2.1) and (2.2) we obtain

$$
\sqrt{z_{1} z_{3}}=k(2 p+1) \text {. }
$$

Berndt, Bhargava and Garvan [3, Lemma 2.1, p. 4168] (see also Berndt [2, Lemma 2.1, p. 94]) have shown that

$$
\begin{aligned}
& a(q)=\sqrt{z_{1} z_{3}} \frac{\left(m^{2}+6 m-3\right)}{4 m}, \\
& b(q)=\sqrt{z_{1} z_{3}} \frac{(3-m)\left(9-m^{2}\right)^{1 / 3}}{4 m^{2 / 3}}, \\
& c(q)=\sqrt{z_{1} z_{3}} \frac{3(m+1)\left(m^{2}-1\right)^{1 / 3}}{4 m} .
\end{aligned}
$$

Theorem 1 now follows on using (2.3) and (2.4) in (2.5)-(2.7).

3. Proof of Theorem 2. Berndt, Bhargava and Garvan [3, eqn. (5.16), p. 4184] (see also Berndt [2, eqn. (5.16), p. 112]) have shown (with $q^{2}$ replaced by $q$ ) that

$$
a(q)=\frac{1}{3}\left(\frac{c^{2}(q)}{c\left(q^{2}\right)}+4 \frac{c^{2}\left(q^{2}\right)}{c(q)}\right) .
$$

Using the expressions for $a(q)$ and $c(q)$ given in Theorem 1 in (3.1), we obtain

$$
\left(1+4 p+p^{2}\right) k=\frac{2^{-2 / 3} 3\left(p(1+p)^{4}\right)^{2 / 3} k^{2}}{c\left(q^{2}\right)}+\frac{2^{7 / 3} 3^{-2} c^{2}\left(q^{2}\right)}{\left(p(1+p)^{4}\right)^{1 / 3} k} .
$$

If we set

$$
x=x(q)=\frac{c\left(q^{2}\right)}{2^{-2 / 3} 3(p(1+p))^{2 / 3} k},
$$

then equation (3.2) becomes after rearrangement

$$
2 p x^{3}-\left(1+4 p+p^{2}\right) x+\left(1+2 p+p^{2}\right)=0 .
$$

Solving the cubic equation (3.4) for $x$, we find

$$
x=1, \frac{1}{2}\left(\sqrt{2 p+5+\frac{2}{p}}-1\right) \text { or }-\frac{1}{2}\left(\sqrt{2 p+5+\frac{2}{p}}+1\right) .
$$


From (1.17) and (1.18) we see that as $q \rightarrow 0^{+}$we have $p \rightarrow 0^{+}$and $k \rightarrow 1^{-}$. Thus from (3.5) we deduce

$$
\lim _{q \rightarrow 0^{+}} x=1,+\infty \text { or }-\infty
$$

respectively. From Theorem 1 and (3.3) we obtain

$$
x=3(1+p)^{2} k \frac{c\left(q^{2}\right)}{c^{2}(q)} .
$$

Hence by (1.19) we have

$$
\lim _{q \rightarrow 0^{+}} x=1 .
$$

From (3.5), (3.6) and (3.8) we deduce that $x=1$. Hence by (3.3) we have

$$
c\left(q^{2}\right)=2^{-2 / 3} 3(p(1+p))^{2 / 3} k
$$

as asserted.

Now Borwein, Borwein and Garvan [5, eqn. (2.28), p. 44] have shown that

$$
a(q)=\frac{1}{2} \frac{c^{2}(q)}{c\left(q^{2}\right)}-\frac{1}{2} \frac{b^{2}(q)}{b\left(q^{2}\right)} .
$$

Appealing to Theorem 1 for the values of $a(q), b(q)$ and $c(q)$, and (3.9) for the value of $c\left(q^{2}\right)$, from (3.10) we obtain

$$
b\left(q^{2}\right)=2^{-2 / 3}((1-p)(1+2 p)(2+p))^{2 / 3} k .
$$

Then, from the identity

$$
a(q) a\left(q^{2}\right)=b(q) b\left(q^{2}\right)+c(q) c\left(q^{2}\right)
$$

(see [5, Theorem 2.6, p. 40]), we obtain

$$
a\left(q^{2}\right)=\left(1+p+p^{2}\right) k .
$$

4. Proof of Theorem 3. The following two identities are proved in [5, Lemma 2.1(ii), p. 36 and eqn. (2.1), p. 37]:

$$
\begin{aligned}
& a\left(q^{3}\right)=\frac{1}{3} a(q)+\frac{2}{3} b(q), \\
& c\left(q^{3}\right)=\frac{1}{3} a(q)-\frac{1}{3} b(q) .
\end{aligned}
$$

The values of $a\left(q^{3}\right)$ and $c\left(q^{3}\right)$ then follow by Theorem 1 .

5. Proof of Theorem 4. Berndt, Bhargava and Garvan [3, eqn. (5.16), p. 4184] (see also Berndt [2, eqn. (5.16), p. 112]) have shown that

$$
a\left(q^{2}\right)=\frac{1}{3}\left(\frac{c^{2}\left(q^{2}\right)}{c\left(q^{4}\right)}+4 \frac{c^{2}\left(q^{4}\right)}{c\left(q^{2}\right)}\right) .
$$


Using the values of $a\left(q^{2}\right)$ and $c\left(q^{2}\right)$ from Theorem 2 in (5.1), we obtain

$$
\begin{aligned}
3\left(1+p+p^{2}\right) k= & \frac{2^{-4 / 3} 3^{2}(p(1+p))^{4 / 3} k^{2}}{c\left(q^{4}\right)} \\
& +2^{8 / 3} 3^{-1}(p(1+p))^{-2 / 3} k^{-1} c^{2}\left(q^{4}\right) .
\end{aligned}
$$

Set

$$
y=y(q)=\frac{c\left(q^{4}\right)}{2^{-4 / 3} 3\left(p^{4}(1+p)\right)^{1 / 3} k} .
$$

Then (5.2) becomes after some rearrangement

$$
p^{2} y^{3}-\left(1+p+p^{2}\right) y+(1+p)=0
$$

Solving the cubic equation (5.4) for $y$, we find

$$
y=1, \frac{1}{p} \text { or } \frac{-(p+1)}{p} .
$$

From (5.3) and (5.5) we deduce

$$
\begin{aligned}
c\left(q^{4}\right)= & 2^{-4 / 3} 3\left(p^{4}(1+p)\right)^{1 / 3} k, 2^{-4 / 3} 3(p(1+p))^{1 / 3} k \\
& \text { or }-2^{-4 / 3} 3\left(p(1+p)^{4}\right)^{1 / 3} k .
\end{aligned}
$$

Berndt, Bhargava and Garvan [3, eqn. (6.3), p. 4188] (see also Berndt [2, eqn. (6.3), p. 116]) have shown that

$$
a(q)-a\left(q^{2}\right)=2 \frac{c^{2}\left(q^{2}\right)}{c(q)} .
$$

Thus

$$
a\left(q^{4}\right)=a\left(q^{2}\right)-2 \frac{c^{2}\left(q^{4}\right)}{c\left(q^{2}\right)} .
$$

From Theorem 2, (5.6) and (5.8), we obtain

$$
a\left(q^{4}\right)=\left(1+p-\frac{1}{2} p^{2}\right) k,\left(-\frac{1}{2}+p+p^{2}\right) k \text { or }\left(-\frac{1}{2}-2 p-\frac{1}{2} p^{2}\right) k .
$$

As $a(0)=1, p(0)=0$ and $k(0)=1$, we deduce that

$$
\begin{aligned}
& a\left(q^{4}\right)=\left(1+p-\frac{1}{2} p^{2}\right) k, \\
& c\left(q^{4}\right)=2^{-4 / 3} 3\left(p^{4}(1+p)\right)^{1 / 3} k .
\end{aligned}
$$

Finally, by (1.5) (with $q$ replaced by $\left.q^{3}\right),(5.9)$ and (5.10), we obtain

$$
b\left(q^{4}\right)^{3}=a\left(q^{4}\right)^{3}-c\left(q^{4}\right)^{3}=2^{-4}(1-p)(1+2 p)(2+p)^{4} k^{3},
$$

so that

$$
b\left(q^{4}\right)=\varepsilon 2^{-4 / 3}\left((1-p)(1+2 p)(2+p)^{4}\right)^{1 / 3} k
$$

for some cube root of unity $\varepsilon$. As $b(0)=1, p(0)=0, k(0)=1$, we must have $\varepsilon=1$. Thus,

$$
b\left(q^{4}\right)=2^{-4 / 3}\left((1-p)(1+2 p)(2+p)^{4}\right)^{1 / 3} k .
$$


6. Proof of Theorem 5. Replacing $q$ by $q^{2}$ in (4.1) and (4.2), we have

$$
\begin{aligned}
& a\left(q^{6}\right)=\frac{1}{3} a\left(q^{2}\right)+\frac{2}{3} b\left(q^{2}\right), \\
& c\left(q^{6}\right)=\frac{1}{3} a\left(q^{2}\right)-\frac{1}{3} b\left(q^{2}\right) .
\end{aligned}
$$

Appealing to Theorem 2 for the values of $a\left(q^{2}\right)$ and $b\left(q^{2}\right)$, we obtain the values of $a\left(q^{6}\right)$ and $c\left(q^{6}\right)$.

7. Proof of Theorem 7. Replacing $q$ by $q^{2}$ in (6.1) and (6.2), we have

$$
\begin{aligned}
& a\left(q^{12}\right)=\frac{1}{3} a\left(q^{4}\right)+\frac{2}{3} b\left(q^{4}\right), \\
& c\left(q^{12}\right)=\frac{1}{3} a\left(q^{4}\right)-\frac{1}{3} b\left(q^{4}\right) .
\end{aligned}
$$

Appealing to Theorem 4 for the values of $a\left(q^{4}\right)$ and $b\left(q^{4}\right)$, we obtain the values of $a\left(q^{12}\right)$ and $c\left(q^{12}\right)$.

8. Proof of Theorem 8. This theorem follows from Theorems 1 and 4 and the relations

$$
\begin{aligned}
a(q)+a(-q) & =2 a\left(q^{4}\right), \\
b(q)+b(-q) & =2 b\left(q^{4}\right), \\
c(q)+c(-q) & =2 c\left(q^{4}\right),
\end{aligned}
$$

proved in [5, p. 40].

9. Proof of Theorem 9. For convenience we set

$$
p_{1}=p\left(q^{2}\right), \quad k_{1}=k\left(q^{2}\right) .
$$

By Theorems 1 and 2 we have

$$
\left(1+4 p_{1}+p_{1}^{2}\right) k_{1}=\left(1+p+p^{2}\right) k
$$

and by Theorems 2 and 4 we have

$$
\left(1+p_{1}+p_{1}^{2}\right) k_{1}=\left(1+p-\frac{1}{2} p^{2}\right) k .
$$

From (9.2) and (9.3) we deduce

$$
\frac{1+4 p_{1}+p_{1}^{2}}{1+p_{1}+p_{1}^{2}}=\frac{1+p+p^{2}}{1+p-\frac{1}{2} p^{2}} .
$$

Writing (9.4) as a quadratic equation in $p_{1}$, we obtain

$$
p^{2} p_{1}^{2}-2\left(1+p-p^{2}\right) p_{1}+p^{2}=0 .
$$

Solving (9.5) for $p_{1}$ gives

$$
p_{1}=\frac{1+p-p^{2}+\lambda((1-p)(1+p)(1+2 p))^{1 / 2}}{p^{2}},
$$

where $\lambda= \pm 1$. Taking $q=0$ in

$$
p^{2} p_{1}=1+p-p^{2}+\lambda((1-p)(1+p)(1+2 p))^{1 / 2},
$$


we obtain, as $p(0)=p_{1}(0)=0,0=1+\lambda$ so that $\lambda=-1$. Hence

$$
p_{1}=p\left(q^{2}\right)=\frac{1+p-p^{2}-((1-p)(1+p)(1+2 p))^{1 / 2}}{p^{2}}
$$

as claimed. Then, from (9.2) and (9.7), we obtain

$$
k_{1}=k\left(q^{2}\right)=\frac{\left(1+p-p^{2}+((1-p)(1+p)(1+2 p))^{1 / 2}\right) k}{2} .
$$

10. Proof of Theorem 11. For convenience we set

$$
p_{2}=p(-q), \quad k_{2}=k(-q) .
$$

By Theorems 2 and 4 we have

$$
\begin{aligned}
\left(1+p_{2}+p_{2}^{2}\right) k_{2} & =\left(1+p+p^{2}\right) k, \\
\left(1+p_{2}-\frac{1}{2} p_{2}^{2}\right) k_{2} & =\left(1+p-\frac{1}{2} p^{2}\right) k .
\end{aligned}
$$

From (10.2) and (10.3) we deduce

$$
\frac{1+p_{2}+p_{2}^{2}}{1+p_{2}-\frac{1}{2} p_{2}^{2}}=\frac{1+p+p^{2}}{1+p-\frac{1}{2} p^{2}}
$$

Rewriting (10.4) as a quadratic equation in $p_{2}$, we obtain

$$
(1+p) p_{2}^{2}-p^{2} p_{2}-p^{2}=0 .
$$

Hence $p_{2}=p$ or $-p /(1+p)$. From (1.17) we have

$$
\begin{aligned}
p & =p(q)=2 q+2 q^{2}+O\left(q^{3}\right), \\
p_{2} & =p(-q)=-2 q+2 q^{2}+O\left(q^{3}\right),
\end{aligned}
$$

for small $|q|$, so that $p_{2} \neq p$. Hence

$$
p_{2}=p(-q)=\frac{-p}{1+p} .
$$

Then, from (10.2) and (10.6), we have

$$
k(-q)=k_{2}=\frac{\left(1+p+p^{2}\right) k}{1-\frac{p}{1+p}+\frac{p^{2}}{(1+p)^{2}}}=(1+p)^{2} k .
$$

11. Proof of Theorem 10. For convenience we set

$$
p_{3}=p\left(q^{3}\right), \quad k_{3}=k\left(q^{3}\right) .
$$


From Theorems $1-5$ and 7 we deduce that

$$
\begin{aligned}
& \left(1+4 p_{3}+p_{3}^{2}\right) k_{3} \\
& \quad=\frac{1}{3}\left(1+4 p+p^{2}+2^{2 / 3}\left((1-p)^{4}(1+2 p)(2+p)\right)^{1 / 3}\right) k \\
& \left(1+p_{3}+p_{3}^{2}\right) k_{3} \\
& \quad=\frac{1}{3}\left(1+p+p^{2}+2^{1 / 3}((1-p)(1+2 p)(2+p))^{2 / 3}\right) k \\
& \left(1+p_{3}-\frac{1}{2} p_{3}^{2}\right) k_{3} \\
& \quad=\frac{1}{3}\left(1+p-\frac{1}{2} p^{2}+2^{-1 / 3}\left((1-p)(1+2 p)(2+p)^{4}\right)^{1 / 3}\right) k
\end{aligned}
$$

Set

$$
X=((1-p)(1+2 p)(2+p))^{1 / 3} .
$$

Then

$$
\begin{aligned}
k_{3}= & -\frac{1}{3}\left(1+4 p_{3}+p_{3}^{2}\right) k_{3}+\frac{2}{3}\left(1+p_{3}+p_{3}^{2}\right) k_{3}+\frac{2}{3}\left(1+p_{3}-\frac{1}{2} p_{3}^{2}\right) k_{3} \\
= & -\frac{1}{9}\left(1+4 p+p^{2}+2^{2 / 3}(1-p) X\right) k+\frac{2}{9}\left(1+p+p^{2}+2^{1 / 3} X^{2}\right) k \\
& +\frac{2}{9}\left(1+p-\frac{1}{2} p^{2}+2^{-1 / 3}(2+p) X\right) k
\end{aligned}
$$

so that

$$
k\left(q^{3}\right)=k_{3}=\frac{1}{9}\left(3+2^{2 / 3}(1+2 p) X+2^{4 / 3} X^{2}\right) k
$$

as asserted.

Next, from (11.2) and (11.3), we deduce

$$
\begin{aligned}
p_{3} k_{3} & =\frac{1}{3}\left(1+4 p_{3}+p_{3}^{2}\right) k_{3}-\frac{1}{3}\left(1+p_{3}+p_{3}^{2}\right) k_{3} \\
& =\frac{1}{9}\left(1+4 p+p^{2}+2^{2 / 3}(1-p) X\right) k-\frac{1}{9}\left(1+p+p^{2}+2^{1 / 3} X^{2}\right) k
\end{aligned}
$$

so that

$$
p_{3} k_{3}=\frac{1}{9}\left(3 p+2^{2 / 3}(1-p) X-2^{1 / 3} X^{2}\right) k .
$$

Hence, from (11.5) and (11.6), we obtain

$$
\begin{aligned}
p\left(q^{3}\right) & =p_{3}=\frac{3 p+2^{2 / 3}(1-p) X-2^{1 / 3} X^{2}}{3+2^{2 / 3}(1+2 p) X+2^{4 / 3} X^{2}} \\
& =\frac{1}{3}\left(-4-3 p+6 p^{2}+4 p^{3}+2^{2 / 3}\left(1-2 p-2 p^{2}\right) X+2^{1 / 3}(1+2 p) X^{2}\right)
\end{aligned}
$$

as claimed.

12. Proof of Theorem 6. Set

$$
t=((1-p)(1+p)(1+2 p))^{1 / 2}
$$

so

$$
t^{2}=1+2 p-p^{2}-2 p^{3} .
$$


By Theorem 11 we have

$$
p\left(q^{2}\right)=\frac{1+p-p^{2}-t}{p^{2}}, \quad k\left(q^{2}\right)=\frac{\left(1+p-p^{2}+t\right) k}{2} .
$$

Thus, by (12.2) and (12.3), we have

$$
p^{2}\left(q^{2}\right)=\frac{\left(2+4 p-2 p^{2}-4 p^{3}+p^{4}\right)-2\left(1+p-p^{2}\right) t}{p^{4}} .
$$

Then, by Theorem 4, we obtain

$$
\begin{aligned}
a\left(q^{8}\right) & =\left(1+p\left(q^{2}\right)-\frac{1}{2} p^{2}\left(q^{2}\right)\right) k\left(q^{2}\right) \\
& =\frac{1}{4}\left(1+p+p^{2}+3((1-p)(1+p)(1+2 p))^{1 / 2}\right) k
\end{aligned}
$$

as asserted.

13. The Eisenstein series $L(q)$. The Eisenstein series $L(q)$ is defined by

$$
L(q)=1-24 \sum_{n=1}^{\infty} \sigma(n) q^{n}
$$

It is shown in $[1$, eqns. (3.84) and (3.87)] that

$$
\begin{aligned}
& L(q)-2 L\left(q^{2}\right)=-\left(1+14 p+24 p^{2}+14 p^{3}+p^{4}\right) k^{2}, \\
& L(q)-3 L\left(q^{3}\right)=-2\left(1+8 p+18 p^{2}+8 p^{3}+p^{4}\right) k^{2},
\end{aligned}
$$

with $p$ and $k$ as defined in (1.8) and (1.9). Applying the triplication principle (Theorem 10) to (13.2) and the duplication principle (Theorem 9) to (13.3), we obtain

$$
\begin{aligned}
& L\left(q^{3}\right)-2 L\left(q^{6}\right)=-\left(1+2 p+2 p^{3}+p^{4}\right) k^{2}, \\
& L\left(q^{2}\right)-3 L\left(q^{6}\right)=-2\left(1+2 p+3 p^{2}+2 p^{3}+p^{4}\right) k^{2}
\end{aligned}
$$

in agreement with [1, eqns. (3.85) and (3.88)]. Applying the triplication principle to (13.4) and the duplication principle to (13.5), we have

$$
\begin{gathered}
\text { (13.6) } L\left(q^{9}\right)-2 L\left(q^{18}\right) \\
=\frac{1}{27}\left(-11-10 p+24 p^{2}-10 p^{3}-11 p^{4}\right. \\
\quad-2^{2 / 3} 4\left(1-p^{3}\right)((1-p)(1+2 p)(2+p))^{1 / 3} \\
\left.\quad-2^{1 / 3} 4\left(1+4 p+p^{2}\right)((1-p)(1+2 p)(2+p))^{2 / 3}\right) k^{2}, \\
(13.7) \quad L\left(q^{4}\right)-3 L\left(q^{12}\right)=\left(-2-4 p+2 p^{3}-\frac{1}{2} p^{4}\right) k^{2} .
\end{gathered}
$$

These results will be needed in the following sections. 
14. The sum $L_{1,2}(q)$. We define

$$
L_{1,2}(q):=\sum_{\substack{n=1 \\ n \equiv 1(\bmod 2)}}^{\infty} \sigma(n) q^{n} .
$$

In this section we evaluate $L_{1,2}(q)$ in terms of $p$ and $k$. We begin by recalling (13.2). We have

$$
L(q)-2 L\left(q^{2}\right)=-\left(1+14 p+24 p^{2}+14 p^{3}+p^{4}\right) k^{2} .
$$

Applying the change of sign principle (Theorem 11) to (14.2) we have

$$
L(-q)-2 L\left(q^{2}\right)=-\left(1-10 p-12 p^{2}-4 p^{3}-2 p^{4}\right) k^{2} .
$$

Subtracting (14.2) from (14.3), we obtain

$$
L(-q)-L(q)=3\left(8 p+12 p^{2}+6 p^{3}+p^{4}\right) k^{2} .
$$

Hence

$$
\begin{aligned}
L_{1,2}(q) & =\sum_{\substack{n=1 \\
n \equiv 1(\bmod 2)}}^{\infty} \sigma(n) q^{n}=\frac{1}{2} \sum_{n=1}^{\infty} \sigma(n) q^{n}-\frac{1}{2} \sum_{n=1}^{\infty} \sigma(n)(-q)^{n} \\
& =\frac{1}{48}(L(-q)-L(q)),
\end{aligned}
$$

so that by $(14.4)$,

$$
L_{1,2}(q)=\left(\frac{1}{2} p+\frac{3}{4} p^{2}+\frac{3}{8} p^{3}+\frac{1}{16} p^{4}\right) k^{2} .
$$

Applying the triplication principle (Theorem 10) to (14.5), we obtain

$$
L_{1,2}\left(q^{3}\right)=\left(\frac{1}{8} p^{3}+\frac{1}{16} p^{4}\right) k^{2} .
$$

15. The sums $L_{1,3}(q)$ and $L_{2,3}(q)$. We define

$$
L_{1,3}(q):=\sum_{\substack{n=1 \\ n \equiv 1(\bmod 3)}}^{\infty} \sigma(n) q^{n}, \quad L_{2,3}(q):=\sum_{\substack{n=1 \\ n \equiv 2(\bmod 3)}}^{\infty} \sigma(n) q^{n} .
$$

These sums have been studied in [12]. In this section we evaluate them in terms of $p$ and $k$. From [12, Theorem 1.4] we have

$$
\begin{aligned}
& L_{1,3}(q)=\left(1+(1-x)^{1 / 3}-2(1-x)^{2 / 3}\right) \frac{w^{2}}{27}, \\
& L_{2,3}(q)=\left(1-2(1-x)^{1 / 3}+(1-x)^{2 / 3}\right) \frac{w^{2}}{27},
\end{aligned}
$$


where, in the notation of [1], we have

$$
\begin{aligned}
& x=x_{3}(q)=B=\frac{27 p(1+p)^{4}}{2\left(1+4 p+p^{2}\right)^{3}}, \\
& w=w_{3}\left(x_{3}(q)\right)=X=\left(1+4 p+p^{2}\right) k .
\end{aligned}
$$

Now

$$
1-x=1-\frac{27 p(1+p)^{4}}{2\left(1+4 p+p^{2}\right)^{3}}=\frac{(1-p)^{4}(1+2 p)(2+p)}{2\left(1+4 p+p^{2}\right)^{3}},
$$

SO

$$
(1-x)^{1 / 3}=\frac{2^{-1 / 3}(1-p)((1-p)(1+2 p)(2+p))^{1 / 3}}{1+4 p+p^{2}} .
$$

Hence

$$
\begin{aligned}
L_{1,3}(q)= & \frac{1}{27}\left(1+8 p+18 p^{2}+8 p^{3}+p^{4}\right. \\
& +2^{-1 / 3}\left(1+3 p-3 p^{2}-p^{3}\right)((1-p)(1+2 p)(2+p))^{1 / 3} \\
& \left.-2^{1 / 3}\left(1-2 p+p^{2}\right)((1-p)(1+2 p)(2+p))^{2 / 3}\right) k^{2}, \\
L_{2,3}(q)= & \frac{1}{27}\left(1+8 p+18 p^{2}+8 p^{3}+p^{4}\right. \\
& -2^{2 / 3}\left(1+3 p-3 p^{2}-p^{3}\right)((1-p)(1+2 p)(2+p))^{1 / 3} \\
& \left.+2^{-2 / 3}\left(1-2 p+p^{2}\right)((1-p)(1+2 p)(2+p))^{2 / 3}\right) k^{2} .
\end{aligned}
$$

From (15.8) and (15.9) we deduce

$$
\begin{aligned}
L_{1,3}(q)+ & 2 L_{2,3}(q)=\frac{1}{9}\left(1+8 p+18 p^{2}+8 p^{3}+p^{4}\right) k^{2} \\
& -\frac{1}{9} \cdot 2^{-1 / 3}\left(1+3 p-3 p^{2}-p^{3}\right)((1-p)(1+2 p)(2+p))^{1 / 3} k^{2}
\end{aligned}
$$

so that

$$
\begin{aligned}
\frac{1}{3} \cdot 2^{2 / 3}\left(1+3 p-3 p^{2}-p^{3}\right)((1-p)(1+2 p)(2+p))^{1 / 3} k^{2} \\
=\frac{2}{3}\left(1+4 p+p^{2}\right)^{2} k^{2}-6 L_{1,3}(q)-12 L_{2,3}(q) .
\end{aligned}
$$

Applying the duplication principle (Theorem 9) to (15.9), we obtain

$$
\begin{aligned}
L_{2,3}\left(q^{2}\right)= & \frac{1}{27}\left(1+2 p+3 p^{2}+2 p^{3}+p^{4}\right. \\
& +2^{-4 / 3}\left(2+3 p-3 p^{2}-2 p^{3}\right)((1-p)(1+2 p)(2+p))^{1 / 3} \\
& \left.-2^{1 / 3}\left(1+p+p^{2}\right)((1-p)(1+2 p)(2+p))^{2 / 3}\right) k^{2} .
\end{aligned}
$$

From (15.8) and (15.11) we deduce

$$
\begin{aligned}
L_{1,3}(q)-2 & L_{2,3}\left(q^{2}\right)=\frac{1}{27}\left(-1+4 p+12 p^{2}+4 p^{3}-p^{4}\right. \\
& -2^{-1 / 3}\left(1-p^{3}\right)((1-p)(1+2 p)(2+p))^{1 / 3} \\
& \left.+2^{1 / 3}\left(1+4 p+p^{2}\right)((1-p)(1+2 p)(2+p))^{2 / 3}\right) k^{2} .
\end{aligned}
$$


From (13.6) and (15.12) we obtain

$$
\begin{aligned}
2^{2 / 3}\left(1-p^{3}\right)( & (1-p)(1+2 p)(2+p))^{1 / 3} k^{2} \\
= & \left(-\frac{5}{2}+p+12 p^{2}+p^{3}-\frac{5}{2} p^{4}\right) k^{2} \\
& \quad-\frac{9}{2} L\left(q^{9}\right)+9 L\left(q^{18}\right)-18 L_{1,3}(q)+36 L_{2,3}\left(q^{2}\right), \\
2^{1 / 3}\left(1+4 p+p^{2}\right) & ((1-p)(1+2 p)(2+p))^{2 / 3} k^{2} \\
= & \left(-\frac{1}{4}-\frac{7}{2} p-6 p^{2}-\frac{7}{2} p^{3}-\frac{1}{4} p^{4}\right) k^{2} \\
& -\frac{9}{4} L\left(q^{9}\right)+\frac{9}{2} L\left(q^{18}\right)+18 L_{1,3}(q)-36 L_{2,3}\left(q^{2}\right) .
\end{aligned}
$$

16. Proof of Theorem 12. By (1.12), Theorem 1 and (13.3), we have

$$
\begin{aligned}
\sum_{n=0}^{\infty} N(1,1 ; n) q^{n} & =a(q)^{2}=\left(1+8 p+18 p^{2}+8 p^{3}+p^{4}\right) k^{2} \\
& =-\frac{1}{2}\left(L(q)-3 L\left(q^{3}\right)\right)=1+\sum_{n=1}^{\infty}(12 \sigma(n)-36 \sigma(n / 3)) q^{n} .
\end{aligned}
$$

Equating coefficients of $q^{n}(n \in \mathbb{N})$ we obtain $N(1,1 ; n)$.

17. Proof of Theorem 13. By (1.12), Theorem 1, Theorem 2, (13.2) and (13.4), we have

$$
\begin{aligned}
\sum_{n=0}^{\infty} N(1,2 ; n) q^{n} & =\left(1+4 p+p^{2}\right)\left(1+p+p^{2}\right) k^{2} \\
& =\frac{1}{4}\left(1+14 p+24 p^{2}+14 p^{3}+p^{4}\right) k^{2}+\frac{3}{4}\left(1+2 p+2 p^{3}+p^{4}\right) k^{2} \\
& =-\frac{1}{4}\left(L(q)-2 L\left(q^{2}\right)\right)-\frac{3}{4}\left(L\left(q^{3}\right)-2 L\left(q^{6}\right)\right) \\
& =1+\sum_{n=1}^{\infty}(6 \sigma(n)-12 \sigma(n / 2)+18 \sigma(n / 3)-36 \sigma(n / 6)) q^{n}
\end{aligned}
$$

Equating coefficients of $q^{n}(n \in \mathbb{N})$ we obtain $N(1,2 ; n)$.

18. Proof of Theorem 14. By (1.12), Theorems 1, 3, and the proof of Theorem 12, we obtain

$$
\begin{aligned}
\sum_{n=0}^{\infty} N(1,3 ; n) q^{n}= & \frac{1}{3}\left(1+4 p+p^{2}\right)^{2} k^{2} \\
& +\frac{1}{3} \cdot 2^{2 / 3}\left(1+3 p-3 p^{2}-p^{3}\right)((1-p)(1+2 p)(2+p))^{1 / 3} k^{2} \\
= & \left(1+4 p+p^{2}\right)^{2} k^{2}-6 L_{1,3}(q)-12 L_{2,3}(q) \\
= & a(q)^{2}-6 L_{1,3}(q)-12 L_{2,3}(q) \\
= & -\frac{1}{2}\left(L(q)-3 L\left(q^{3}\right)\right)-6 L_{1,3}(q)-12 L_{2,3}(q)
\end{aligned}
$$


that is,

$$
\begin{aligned}
\sum_{n=0}^{\infty} N(1,3 ; n) q^{n}= & +\sum_{n=1}^{\infty}(12 \sigma(n)-36 \sigma(n / 3)) q^{n} \\
& -6 \sum_{\substack{n=1 \\
n \equiv 1(\bmod 3)}}^{\infty} \sigma(n) q^{n}-12 \sum_{\substack{n=1 \\
n \equiv 2(\bmod 3)}}^{\infty} \sigma(n) q^{n}
\end{aligned}
$$

Equating coefficients of $q^{n}(n \in \mathbb{N})$ we obtain $N(1,3 ; n)$.

19. Proof of Theorem 15. By (1.12), Theorems 1 and 4, (13.7), (14.5) and (14.6), we obtain

$$
\begin{aligned}
\sum_{n=0}^{\infty} N(1,4 ; n) q^{n}= & -\frac{1}{2}\left(-2-4 p+2 p^{3}-\frac{1}{2} p^{4}\right) k^{2} \\
& +6\left(\frac{1}{2} p+\frac{3}{4} p^{2}+\frac{3}{8} p^{3}+\frac{1}{16} p^{4}\right) k^{2}-18\left(\frac{1}{8} p^{3}+\frac{1}{16} p^{4}\right) k^{2} \\
= & -\frac{1}{2}\left(L\left(q^{4}\right)-3 L\left(q^{12}\right)\right)+6 L_{1,2}(q)-18 L_{1,2}\left(q^{3}\right) \\
= & 1+12 \sum_{n=1}^{\infty} \sigma(n / 4) q^{n}-36 \sum_{n=1}^{\infty} \sigma(n / 12) q^{n} \\
& +6 \sum_{n=1}^{\infty} \sigma(n) q^{n}-18 \sum_{n=1}^{\infty} \sigma(n / 3) q^{n} . \\
n \equiv 1(\bmod 2) &
\end{aligned}
$$

Equating coefficients of $q^{n}(n \in \mathbb{N})$ we obtain $N(1,4 ; n)$.

20. Proof of Theorem 16. Appealing to (1.12), Theorems 1 and 5, (15.14) and (13.4), we obtain

$$
\begin{aligned}
\sum_{n=0}^{\infty} N(1,6 ; n) q^{n}= & \frac{1}{3}\left(\left(1+4 p+p^{2}\right)\left(1+p+p^{2}\right)\right. \\
& \left.+2^{1 / 3}\left(1+4 p+p^{2}\right)((1-p)(1+2 p)(2+p))^{2 / 3}\right) k^{2} \\
= & \frac{1}{3}\left(\left(1+5 p+6 p^{2}+5 p^{3}+p^{4}\right) k^{2}\right. \\
& \left.+2^{1 / 3}\left(1+4 p+p^{2}\right)((1-p)(1+2 p)(2+p))^{2 / 3} k^{2}\right) \\
= & \frac{1}{3}\left(\left(1+5 p+6 p^{2}+5 p^{3}+p^{4}\right) k^{2}\right. \\
& +\left(-\frac{1}{4}-\frac{7}{2} p-6 p^{2}-\frac{7}{2} p^{3}-\frac{1}{4} p^{4}\right) k^{2} \\
& \left.-\frac{9}{4} L\left(q^{9}\right)+\frac{9}{2} L\left(q^{18}\right)+18 L_{1,3}(q)-36 L_{2,3}\left(q^{2}\right)\right)
\end{aligned}
$$




$$
\begin{aligned}
= & \left(\frac{1}{4}+\frac{1}{2} p+\frac{1}{2} p^{3}+\frac{1}{4} p^{4}\right) k^{2}-\frac{3}{4} L\left(q^{9}\right)+\frac{3}{2} L\left(q^{18}\right) \\
& +6 L_{1,3}(q)-12 L_{2,3}\left(q^{2}\right) \\
= & -\frac{1}{4}\left(L\left(q^{3}\right)-2 L\left(q^{6}\right)\right)-\frac{3}{4} L\left(q^{9}\right)+\frac{3}{2} L\left(q^{18}\right) \\
& +6 L_{1,3}(q)-12 L_{2,3}\left(q^{2}\right) \\
= & +\sum_{n=1}^{\infty}(6 \sigma(n / 3)-12 \sigma(n / 6)+18 \sigma(n / 9)-36 \sigma(n / 18)) q^{n} \\
& +6 \sum_{n=1}^{\infty} \sigma(n) q^{n}-12 \sum_{n=1}^{\infty} \sigma(n / 2) q^{n} . \\
n \equiv 1(\bmod 3) &
\end{aligned}
$$

Equating coefficients of $q^{n}(n \in \mathbb{N})$ we obtain

$$
N(1,6 ; n)= \begin{cases}6 \sigma(n / 3)-12 \sigma(n / 6)+18 \sigma(n / 9)-36 \sigma(n / 18) & \text { if } n \equiv 0(\bmod 3) \\ 6 \sigma(n)-12 \sigma(n / 2) & \text { if } n \equiv 1(\bmod 3) \\ 0 & \text { if } n \equiv 2(\bmod 3)\end{cases}
$$

When $n \equiv 0(\bmod 3)$ we have the elementary identities

$$
\begin{gathered}
\sigma(n)=4 \sigma(n / 3)-3 \sigma(n / 9), \\
\sigma(n / 2)=4 \sigma(n / 6)-3 \sigma(n / 18),
\end{gathered}
$$

SO

$$
\begin{aligned}
6 \sigma(n / 3)-12 \sigma(n / 6)+ & 18 \sigma(n / 9)-36 \sigma(n / 18) \\
= & -6 \sigma(n)+12 \sigma(n / 2)+30 \sigma(n / 3)-60 \sigma(n / 6),
\end{aligned}
$$

completing the proof.

21. Proof of Theorem 17. Appealing to (1.12), Theorems 2, 3 and (15.13), we obtain

$$
\begin{aligned}
\sum_{n=0}^{\infty} N(2,3 ; n) q^{n}= & \frac{1}{3}\left[\left(1+p+p^{2}\right)\left(1+4 p+p^{2}\right) k^{2}\right. \\
& \left.+2^{2 / 3}\left(1-p^{3}\right)((1-p)(1+2 p)(2+p))^{1 / 3} k^{2}\right] \\
= & \frac{1}{3}\left[\left(1+5 p+6 p^{2}+5 p^{3}+p^{4}\right) k^{2}\right. \\
& +\left(-\frac{5}{2}+p+12 p^{2}+p^{3}-\frac{5}{2} p^{4}\right) k^{2} \\
& \left.-\frac{9}{2} L\left(q^{9}\right)+9 L\left(q^{18}\right)-18 L_{1,3}(q)+36 L_{2,3}\left(q^{2}\right)\right] \\
= & \left(-\frac{1}{2}+2 p+6 p^{2}+2 p^{3}-\frac{1}{2} p^{4}\right) k^{2}-\frac{3}{2} L\left(q^{9}\right)+3 L\left(q^{18}\right) \\
& -6 L_{1,3}(q)+12 L_{2,3}\left(q^{2}\right) .
\end{aligned}
$$


Next

\section{Hence}

$$
\begin{aligned}
\left(-\frac{1}{2}+\right. & \left.2 p+6 p^{2}+2 p^{3}-\frac{1}{2} p^{4}\right) k^{2} \\
= & \frac{1}{4}\left(1+14 p+24 p^{2}+14 p^{3}+p^{4}\right) k^{2}-\frac{3}{4}\left(1+2 p+2 p^{3}+p^{4}\right) k^{2} \\
& =-\frac{1}{4}\left(L(q)-2 L\left(q^{2}\right)\right)+\frac{3}{4}\left(L\left(q^{3}\right)-2 L\left(q^{6}\right)\right) \\
& =-\frac{1}{4} L(q)+\frac{1}{2} L\left(q^{2}\right)+\frac{3}{4} L\left(q^{3}\right)-\frac{3}{2} L\left(q^{6}\right) .
\end{aligned}
$$

$$
\begin{aligned}
\sum_{n=0}^{\infty} N(2,3 ; n) q^{n}= & -\frac{1}{4} L(q)+\frac{1}{2} L\left(q^{2}\right)+\frac{3}{4} L\left(q^{3}\right)-\frac{3}{2} L\left(q^{6}\right)-\frac{3}{2} L\left(q^{9}\right) \\
& +3 L\left(q^{18}\right)-6 L_{1,3}(q)+12 L_{2,3}\left(q^{2}\right) \\
= & 1+\sum_{n=1}^{\infty}(6 \sigma(n)-12 \sigma(n / 2)-18 \sigma(n / 3) \\
& +36 \sigma(n / 6)+36 \sigma(n / 9)-72 \sigma(n / 18)) q^{n} \\
& -6 \sum_{n=1}^{\infty} \sigma(n) q^{n}+12 \sum_{n=1}^{\infty} \sigma(n / 2) q^{n} \\
n \equiv 1(\bmod 3) &
\end{aligned}
$$

Equating coefficients of $q^{n}(n \in \mathbb{N})$ we obtain

$$
N(2,3 ; n)= \begin{cases}6 \sigma(n)-12 \sigma(n / 2)-18 \sigma(n / 3) & \\ \quad+36 \sigma(n / 6)+36 \sigma(n / 9)-72 \sigma(n / 18) & \text { if } n \equiv 0(\bmod 3), \\ 0 & \text { if } n \equiv 1(\bmod 3), \\ 6 \sigma(n)-12 \sigma(n / 2) & \text { if } n \equiv 2(\bmod 3) .\end{cases}
$$

When $n \equiv 0(\bmod 3)$ then from $(20.1)$ and $(20.2)$ we have

$$
\sigma(n / 9)=\frac{4}{3} \sigma(n / 3)-\frac{1}{3} \sigma(n), \quad \sigma(n / 18)=\frac{4}{3} \sigma(n / 6)-\frac{1}{3} \sigma(n / 2),
$$

so that

$$
\begin{aligned}
& 6 \sigma(n)-12 \sigma(n / 2)-18 \sigma(n / 3)+36 \sigma(n / 6)+36 \sigma(n / 9)-72 \sigma(n / 18) \\
&=6 \sigma(n)-12 \sigma(n / 2)-18 \sigma(n / 3)+36 \sigma(n / 6)+48 \sigma(n / 3) \\
&-12 \sigma(n)-96 \sigma(n / 6)+24 \sigma(n / 2) \\
&=-6 \sigma(n)+12 \sigma(n / 2)+30 \sigma(n / 3)-60 \sigma(n / 6),
\end{aligned}
$$

completing the proof.

\section{References}

[1] S. Alaca and K. S. Williams, Evaluation of the convolution sums $\sum_{l+6 m=n} \sigma(l) \sigma(m)$ and $\sum_{2 l+3 m=n} \sigma(l) \sigma(m)$, submitted for publication.

[2] B. C. Berndt, Ramanujan's Notebooks, Part V, Springer, New York, 1998. 
[3] B. C. Berndt, S. Bhargava and F. G. Garvan, Ramanujan's theories of elliptic functions to alternative bases, Trans. Amer. Math. Soc. 347 (1995), 4163-4244.

[4] J. M. Borwein and P. B. Borwein, A cubic counterpart of Jacobi's identity and the AGM, ibid. 323 (1991), 691-701.

[5] J. M. Borwein, P. B. Borwein and F. G. Garvan, Some cubic modular identities of Ramanujan, ibid. 343 (1994), 35-47.

[6] J. G. Huard, Z. M. Ou, B. K. Spearman and K. S. Williams, Elementary evaluation of certain convolution sums involving divisor functions, in: Number Theory for the Millennium, II, M. A. Bennett et al. (eds.), A. K. Peters, Natick, MA, 2002, 229-274.

[7] J. Liouville, Sur la forme $x^{2}+x y+y^{2}+z^{2}+z t+t^{2}$, J. Math. Pures Appl. 8 (1863), 141-144.

[8] -, Sur la forme $x^{2}+x y+y^{2}+2 z^{2}+2 z t+2 t^{2}$, ibid. 8 (1863), 308-310.

[9] - Sur la forme $x^{2}+x y+y^{2}+6 z^{2}+6 z t+6 t^{2}$, ibid. 9 (1864), 181-182.

[10] - Sur la forme $2 x^{2}+2 x y+2 y^{2}+3 z^{2}+3 z t+3 t^{2}$, ibid. 9 (1864), 183-184.

[11] - Sur la forme $x^{2}+x y+y^{2}+3 z^{2}+3 z t+3 t^{2}$, ibid. 9 (1864), 223-224.

[12] K. S. Williams, A cubic transformation formula for ${ }_{2} F_{1}\left(\frac{1}{3}, \frac{2}{3} ; 1 ; z\right)$ and some arithmetic convolution formulae, Math. Proc. Cambridge Philos. Soc. 137 (2004), 519539 .

Centre for Research in Algebra and Number Theory

School of Mathematics and Statistics

Carleton University

Ottawa, Ontario, Canada K1S 5B6

E-mail: aalaca@math.carleton.ca

salaca@math.carleton.ca

williams@math.carleton.ca

Received on 26.2.2006

and in revised form on 10.5.2006 